THU0165

OBESITY AS ONE OF THE COMMODITIES WAS THE ROBUSTEST PREDICTION FACTORS FOR POST THERAPEUTIC CLINICAL REMISSION OF RHEUMATOID ARTHRITIS WITH SHORT DISEASE DURATION RESULTS FROM KANSAI CONSORTIUM FOR WELLBEING OF RHEUMATIC DISEASE PATIENTS

K. Murakami ${ }^{1}$, M. Hashimoto ${ }^{2}$, K. Murata $^{2}$, W. Yamamoto ${ }^{2,3}$, R. Hara $^{4}$

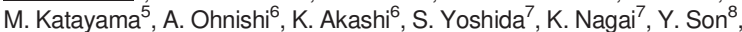
H. Amuro ${ }^{8}$, T. Hirano ${ }^{9}$, K. Ebina ${ }^{10}$, K. Nishitani ${ }^{2}$, M. Tanaka ${ }^{2}$, H. Ito ${ }^{11}$, K. Ohmura ${ }^{1}$ T. Mimori'. ' ${ }^{1}$ Department of Rheumatology and Clinical Immunology; ${ }^{2}$ Department of Advanced Medicine for Rheumatic Diseases, Kyoto University Graduate School of Medicine, Kyoto; ${ }^{3}$ Department of Health Information Management, Kurashiki Sweet Hospital, Kurashiki, ${ }^{4}$ The Center for Rheumatic Diseases, Nara Medical University, Nara; ${ }^{5}$ Department of Rheumatology, Osaka Red Cross Hospital, Osaka; ${ }^{6}$ Department of Rheumatology and Clinical Immunology, Kobe University Graduate School of Medicine, Kobe; ${ }^{7}$ Department of Internal Medicine (IV), Osaka Medical College, Takatsuki; ${ }^{8}$ First Department of Internal Medicine, Kansai Medical University, Hirakata; ${ }^{9}$ Department of Respiratory Medicine, Allergy and Rheumatic Disease; ${ }^{10}$ Department of Orthopaedic Surgery, Osaka University Graduate School of Medicine, Osaka; ${ }^{11}$ Department of Orthopaedic Surgery, Kyoto University Graduate School of Medicine, Kyoto, Japan

Background: A part of rheumatoid arthritis (RA) patients are resistant to clinical remission (CR) irrespective of therapies. In addition to known risk factors, systemic organ complications are assumed to interfere with $\mathrm{CR}$.

Objectives: To extract the predictive comorbidities of clinical response of disease activities.

Methods: In Kansai consortium for well-being of rheumatic disease patients (ANSWER) cohort, which was the real world cohort of clinical database of rheumatic diseases, RA patients within 3 years of disease duration were included and followed. Using logistic regression analysis, background factors at the initial visit were extracted in order to predict CR after 1 year (1 year-non CR).

Results: 651 patients met in the inclusion criteria were under the analysis. Of those, $245(37.6 \%)$ cases were resulted in 1 year-non CR. The average scores of DAS28-CRP at first visit and one year later was 3.51 and 2.02, respectively. Logistic regression analysis revealed that DAS28-CRP at first visit (OR 1.42/unit, $95 \% \mathrm{Cl} 1.24-1.63$ ), concomitant use of methotrexate (MTX) or biologic disease modifying rheumatic drugs (bDMARDs) (OR 2.04, 95\% Cl 1.41-2.96) and body mass index (BMI) (OR 1.07/unit, $95 \% \mathrm{Cl} 1.02-1.12$ ) were significant predictive factors of 1 year-non CR, but not in the case with gender, age, disease duration, hypertension, diabetes, dyslipidemia, lung diseases, heart diseases, digestive tissue diseases, history of malignancy nor concomitant autoimmune diseases. Using propensity score matching (1:1) stratified by gender, age, disease duration, concomitant use of MTX or bDMARDs and DAS28-CRP at baseline, the 49 pairs were matched between obese (defined as body mass index (BMI) over than 28) and non-obese patients. In these cohort, mean score of DAS28-CRP after 1 year was significantly higher in obese patients than in non-obese patients (2.63 and 2.18 , respectively, see the figure 1 ).

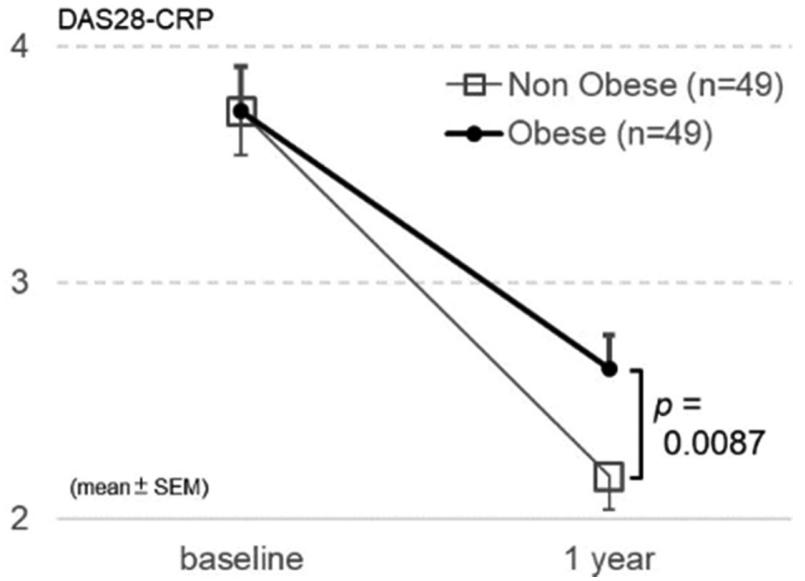

Conclusions: In these early arthritis cohort, obese patients tend to remain higher disease activities even considering with gender, age and therapeutic management, although validation studies should be added to confirm these findings.

Acknowledgements: None.

Disclosure of Interest: None declared

DOI: 10.1136/annrheumdis-2018-eular.1680

\section{THU0166 \\ THE DIASTOLIC DYSFUNCTION IN PATIENTS WITH RHEUMATOID ARTHRITIS}

L. Feiskhanova. Department of hospital therapy, Kazan state medical University, Kazan, Russian Federation

Background: Rheumatoid arthritis is autoimmune rheumatic disease characterised by injury not only the joints but also other organs, including the heart. It is known that the presence of rheumatoid arthritis increases the risk of fatal cardiovascular complications by 1.5 times compared to the general population.

Objectives: to identify features of development of diastolic dysfunction in patients with rheumatoid arthritis.

Methods: we examined 180 patients with rheumatoid arthritis. The activity of disease was defined according to the scale of the DAS-28. By echocardiography in 101 patients (group 1) were identified diastolic dysfunction of the left or both ventricles and in 79 patients (group 2) it was absent. Both groups were matched for age and sex. We determined the following echocardiographic parameters: mitral $E / A$, tricuspid $E / A$, end-diastolic dimension of left ventricle. In addition to echocardiography, patients underwent the vectorcardiography with the assessment of electrophysiological parameters: the squares of loops $\mathrm{P}, \mathrm{QRS}, \mathrm{T}$, maximum vec tor (MV), MV-azimuth and MV- ascent. To compare two independent groups on quantitative grounds used nonparametric methods, the rank correlation and Mann-Whitney test. Differences were considered to be valid when $\mathrm{p}<0.05$.

Results: when comparing the groups revealed that in the 1st group, DAS-28 was higher than in the 2 nd $(\mathrm{p}<0,05): 5,575^{5,17 ; 6,15}$ and $5.32^{4,8 ; 5,8}$ respectively. In the 1 st group, the square of loop QRS and the MV- ascent directly correlated with E/A of mitral valve $(p<0.05)$, whereas in the 2 nd group, we have established a direct relationship with end-diastolic dimension $(p<0.05)$.

Conclusions: the results indicate that increased activity of rheumatoid arthritis contributes to the development of diastolic dysfunction of the myocardium. In addition, the decrease in E/A observed in diastolic dysfunction, accompanied by electrophysiological remodelling and reduction in electrical activity of the myocardium of the left ventricle, diagnosed during registration of vectorcardiogram. Moreover, even in the absence of diastolic dysfunction, a tendency to its development in the presence of electrophysiological remodelling. This demonstrates the relationship between early electrophysiological, structural-geometric changes in patients with rheumatoid arthritis. Early diagnosis allows for timely start prevention of remodelling in patients with rheumatologic diseases.

Disclosure of Interest: None declared

DOI: 10.1136/annrheumdis-2018-eular.1862

\section{THU0167 FALLS IN RHEUMATOID ARTHRITIS (RA) AND ITS RELATION TO DISEASE ACTIVITY, DISABILITY AND PHYSICAL PERFORMANCE TESTS}

J.Z. Gaino ${ }^{1}$, M.B. Bertolo ${ }^{2}$, C.S. Nunes ${ }^{1}$, C.M. Barbosa ${ }^{1}$, Z. Sachetto ${ }^{2}$, M. Davitt ${ }^{1}$ E.D.P. Magalhães ${ }^{2} .{ }^{1}$ UNICAMP, Campinas, Brazil; ${ }^{2}$ Rheumatology, UNICAMP, Campinas, Brazil

Background: Rheumatoid patients(RA)are known to have an increased falls incidence with a threefold increased risk of hip fracture. Few studies have been conducted to evaluate the relation between falls, disease activity, disability and physical functioning.

Objectives: To evaluate the prevalence of falls in RA and its relation with disease activity, disability and physical performance tests.

Methods: 113 RA patients were evaluated from the outpatient clinic of the Rheumatology Division of the State University of Campinas/Unicamp. Patients were assessed for occurrence of falls in last year, fear of falling, sociodemographic and clinical data (medication, visual impairment, vertigo, physical activity, body mass index, disease duration, rheumatoid factor, lower limb swollen and tender joints, foot tactile sensitivity, disease activity-CDAI and disability-HAQ). Subjects were submitted to Berg Balance Scale-BBS, the Timed Up and Go Test-TUG and 5 Time Sit Down-to-Stand Up Test-SST5 and were divided in "Fallers" and "NonFallers" groups. For comparison of groups the chi-squared test, Fisher's exact test and Mann-Whitney were used. Univariate linear regression and multivariate analysis were used to analyse the relation between sociodemographic, clinical data and physical tests with the occurrence of falls. Kruskal- Wallis test was used for analysis of the association of BBS, TUG and SST5 with CDAI and HAQ. The data were analysed with a $5 \%$ level of significance.

Results: $52.21 \%$ reported the occurrence of falls in the past 12 months and $62.8 \%$ were fearful of falling. Comparing "Fallers" and "Non-Fallers", significant differences were noted for $\operatorname{HAQ}(p=0.0242)$, fear of falling $(p=0.0196)$ and BBS $(p=0.0120)$. After univariate logistic regression there was association of falls with income(OR 1.05), HAQ(OR 1.945), fear of falling(OR 2.586) and TUG (OR 1.09). In multivariate model, income was independently linked to falls(OR 1.07). BBS, TUG, SST5 were correlated with CDAI and HAQ $(p<0.05)$. 
Conclusions: RA patients have high prevalence of falls and fear of falling. Income was an independent fall risk factor. BBS seems to be higher among fallers and TUG can be considered a fall risk predictor. BBS, TUG and SST5 are related to CDAI and HAQ. CDAI does not seems to be a good instrument to predict falls due to its variability over time. In clinical practice, HAQ can be a valuable tool to recognise patients with an increased risk of falls.

\section{REFERENCES:}

[1] Stanmore EK, Oldham J, Skelton DA, et al. Risk factors for falls in adults with rheumatoid arthritis: a prospective study. Arthritis Care Res 2013 Aug;65(8):1251-8.

[2] Brenton-Rule A, Dalbeth N, Menz HB, et al. The incidence and risk factors for falls in adults with rheumatoid arthritis: a systematic review. Semin Arthritis Rheum 2014;44:389-98.

[3] Podsiadlo D, Richardson S. The timed "Up \& Go": a test of basic functional mobility for frail elderly persons. J AmGeriatr Soc 1991; 39:142-8.16.

[4] Berg KO, Maki BE, Williams Jl, et al. Clinical and laboratory measures of postural balance in an elderly population. Arch Phys Med Rehabil1992;73:1073-1080.

[5] Bohannon RW. Test-retest reliability of the five-repetition sit-to-stand test: a systematic review of the literature involving adults. J Strength Cond Res 2011; 25:3205-7.

Disclosure of Interest: None declared

DOI: 10.1136/annrheumdis-2018-eular.3558

\section{THU0168 RELATIONSHIPS BETWEEN BODY FAT COMPOSITION ASSESSED WITH BIOELECTRICAL IMPEDANCE ANALYSIS, SERUM ADIPOKINES AND DISEASE ACTIVITY IN PATIENTS WITH RHEUMATOID ARTHRITIS}

M.G. Anelli, V. Venerito, G. Lopalco, F. Cacciapaglia, C. Scioscia, M. Giannotta, G. Righetti, F. Montini, D. Natuzzi, R. Bizzoca, N. Lacarpia, R. Fanizzi,

G. Lapadula, F. lannone. Department of Emergency and Organ Transplantations, Rheumatology Unit, University of Bari "Aldo Moro", Bari, Italy

Background: High-grade inflammation in patients affected with Rheumatoid Arthritis (RA) leads to an imbalanced body composition characterised by increased fat mass and decreased lean mass, with stable or increased body weight, resulting in little or no change in body mass index (BMI). ${ }^{1}$ This condition, known as rheumatoid cachexia, is proven to be reversible once inflammatory process has been shut down. BMl and waist-to-hip ratio (WHR) are used as indirect measurements of visceral fat even if not capable of discriminating it from subcutaneous fat tissue. An alternative method proposed for assessment of fat composition, indicating nutritional status, is bioelectrical impedance analysis (BIA).

Objectives: To analyse body fat composition of patients with RA assessed using either BIA and anthropometric measures, investigating relationships between the related indices, serum adipokines and disease activity.

Methods: The body composition of 87 consecutive patients (72 female, $82.76 \%$ ) affected with RA according to 2010 ACR/EULAR classification criteria, mean age of $52.42 \pm 13.29$, mean disease duration of $10.71 \pm 8.58$ years, treated with DMARDs and/or biologics, was assessed during their visit to our outpatient department. Data including demographic characteristics clinical manifestations, disease activity indices (DAS28-ESR, DAS28-CRP, CDAI, SDAI), Health Assessment Questionnaire (HAQ), lipid profile, as well as radiological findings were collected. Patients underwent to anthropometric measures (WHR and BMI) and BIA for the evaluation of fat-free mass (FFM), fat mass (FM) and the derived indices (fat-free mass index (FFMI) and fat mass index (FMI)). In addition for each patient blood samples were collected to determine serum levels of several adipokines (leptin, adiponectin, visfatin, resistin). Statistical tests as well as linear and logistic regression analysis were carried out.

Results: DAS28-ESR was found to be related to $\mathrm{FMI}(\mathrm{p}=0.02 ; \mathrm{r}=0.22)$ and to $\mathrm{BMI}$ $(p=0.03 ; r=0.22)$. In addition, in patients in whom DAS28-ESR remission was not achieved, FMI but not BMI was found to be related to the latter disease activity index $(p=0.007, r=0.37)$. FMI was shown to correlate to HAQ $(p=0.01, r=0.31)$. Higher serum levels of leptin were found to predict higher FMI and BMI $(p<0.0001$ for both, $r=0.68$ and $r=0.57$, respectively) with DAS28 being also related to the latter adipokine $(\mathrm{p}=0.02 ; \mathrm{r}=0.24)$. FFMI was inversely related to adiponectine serum levels $(p=0.0003 ; r=-0.40)$. Both male and female patients classified as underweight for BMI were found to have a normal mean FFMI, with both groups presenting a mean DAS28-ESR score indicating remission.

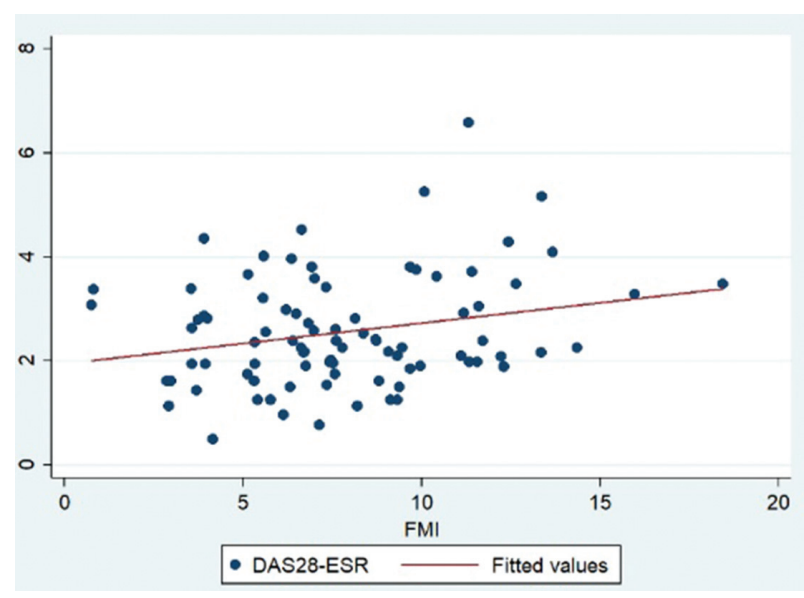

Conclusions: Either body fat composition assessed with BIA-related indices and serum leptin have been noticed to predict disease activity. Moreover nutritional status of patients in remission according to DAS28-ESR may be better assessed by determining indices brought by BIA along with $\mathrm{BMI}$, in order to rule out rheumatoid cachexia on the basis of FMI and FFMI.

\section{REFERENCE:}

[1] Ferraz-Amaro I, Gonzalez-Juanatey C, Lopez-Mejias R, Riancho-Zarrabeitia L, Gonzalez Gay MA. Metabolic syndrome in rheumatoid arthritis. Mediators Inflamm 2013.

Disclosure of Interest: None declared

DOI: 10.1136/annrheumdis-2018-eular.6674

\section{THU0169 RELATIONSHIP OF FRAILTY AND DISEASE ACTIVITY IN PATIENTS WITH RHEUMATOID ARTHRITIS: DATA FROM THE CHIKARA STUDY}

M. Tada ${ }^{1}$, Y. Yamada ${ }^{2}$, K. Mandai ${ }^{2}$, N. Hidaka ${ }^{1}{ }^{1}$ Orthopaedic Surgery, Osaka City General Hospital; ${ }^{2}$ Orthopaedic Surgery, Osaka City University Graduate School of Medicine, Osaka, Japan

Background: Frailty is defined as degradation of physical and cognition function in elderly adult ${ }^{1}$. The characteristics of frailty include not only physical problems as co-morbidity and disability, but also mental and social problems. It is unclear of relationship between frailty and disease activity of patients with rheumatoid arthritis $(\mathrm{RA})$.

Objectives: We investigated the relative factors about frailty in patients with RA from a prospective observational study.

Methods: 95 from 100 patients entered the CHIKARA study (Correlation researcH of sarcopenla, sKeletal muscle and disease Activity in Rheumatoid Arthritis) were investigated by frailty check list (maximal score is 25). According to reported article, frailty was defined from 8 to 25 and pre-frailty was from 4 to 7 , and normal was from 0 to 3 . We investigated relationship of disease activity in frailty, pre-frailty and normal groups, and analysed the relative factors for frailty.

Results: The prevalence of frailty, pre-frailty, and normal was $19 \%, 39 \%$ and $42 \%$, respectively. The character of groups indicated at table 1 . Frailty group was the oldest of three groups. Disease activity sore 28 erythrocyte sedimentation rate (DAS28ESR) and matrix metalloproteinase 3 (MMP3) of frailty group was higher than those of pre-frailty and normal groups. Whereas, modified health assessment questionnaire ( $\mathrm{mHAQ}$ ) of frailty group was lower than those of pre-frailty and normal groups. Normal was $66.6 \%$ and frailty was $6.7 \%$ in remission patients However, Normal was $13.3 \%$ and frailty was $46.7 \%$ in moderate and high disease activity patients (figure 1). The prevalence of frailty was increased with disease activity. The relative factors for frailty were age, locomotive syndrome, DAS28ESR, mHAQ, and Steinbroker class, positively and leg muscle score and grip strength, negatively by univariate analysis. Steinbroker class (odds ratio: 3.25 $95 \% \mathrm{Cl}: 1.11-9.51, \mathrm{p}=0.031$ ) and $\mathrm{mHAQ}$ (odds ratio: $1.29,95 \% \mathrm{Cl}: 1.13-1.46$, $\mathrm{p}<0.001$ ) were independent relative factors by multivariate analysis. 